\title{
$\mathrm{CO}_{2}$ cooling experience ( $\left.\mathrm{LHCb}\right)$
}

\author{
Martin van Beuzekom, Ann Van Lysebetten ${ }^{1}$, Bart Verlaat \\ Nikhef \\ Kruislaan 409, 1098 SJ Amsterdam, The Netherlands \\ E-mail: Ann.Van. Lysebetten@cern.ch
}

The thermal control system of the LHCb Vertex LOcator (VELO) is a two-phase $\mathrm{CO}_{2}$ cooling system based on the 2-Phase Accumulator Controlled Loop (2PACL) method. Liquid carbon dioxide is mechanically pumped in a closed loop, chilled by a water-cooled freon chiller and evaporated in the VELO detector. The main goal of the system is the permanent cooling of the VELO silicon sensors and of the heat producing front-end electronics inside a vacuum environment. This paper describes the design and the performance of the system. First results obtained during commissioning are also presented.

The 16th International Workshop on Vertex detectors

Lake Placid, NY, USA

23-28 September, 2007

\footnotetext{
$1 \quad$ Speaker
} 


\section{LHCb and the Vertex Locator}

The LHCb experiment [1] is dedicated to precision measurements of CP violation in the $\mathrm{B}$ sector and to the search of rare B decays. All angles and some sides of the unitarity triangle are addressed by a multitude of B decay channels for which an efficient trigger system is needed. Powerful particle identification is another requirement set by the physics goals of the experiment.

Two crucial components of the LHCb experiment are high track reconstruction efficiency and efficient vertex identification. The ability to distinguish the primary and secondary vertices on an event by event basis is essential for the LHCb physics program. The Vertex Locator (VELO) [2] has to provide precise measurements of track coordinates close to the interaction region. The VELO detector consists of two halves each equipped with 23 silicon modules along the beam direction. The active part of the silicon starts at a distance of $8 \mathrm{~mm}$ from the beam. The detector is mounted in a setup similar to Roman pots. A design impression, with only a few detector modules installed is given in Figure 1.

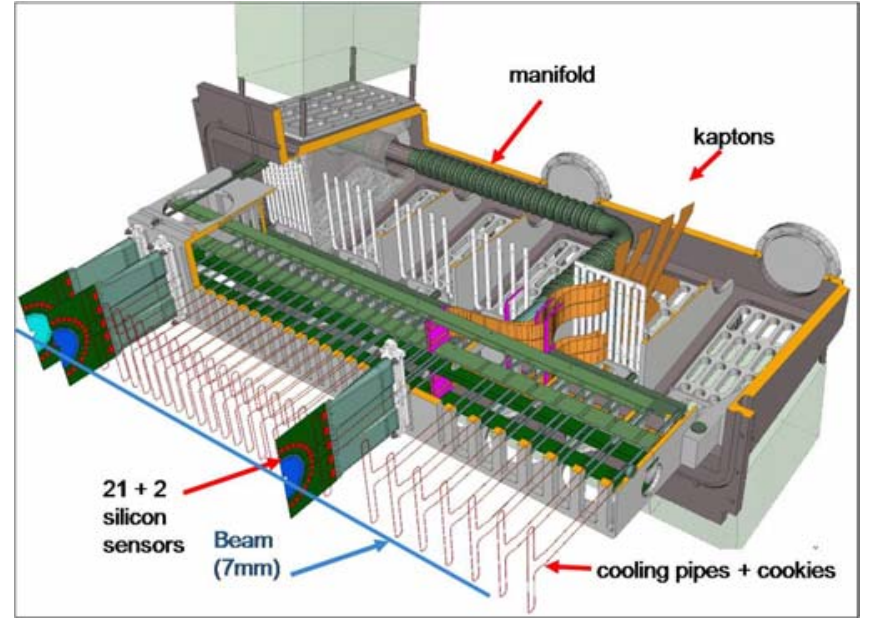

Fig. 1: One of the two VELO detector halves with some modules installed.
The detector will be operated in an extremely harsh radiation environment which is strongly nonuniform. The damage to silicon at the most irradiated area during one year of operation is equivalent to that of $1 \mathrm{MeV}$ neutrons with a fluence of $1.3 \times 10^{14} / \mathrm{cm}^{2}$. The modules need to be cooled in order to hold annealing and prevent thermal runaway of the silicon. The temperature of the silicon sensors is required to be below $0^{\circ} \mathrm{C}$ at all times. As the VELO is operated in vacuum ( $\sim 10^{-4}$ mbar) cooling of the sensors must be achieved by conductance to the refrigerant. A conductance path causes thermal gradients and due to the VELO module layout a cooling temperature of $-25^{\circ} \mathrm{C}$ is required. The refrigerant must be radiation hard, cope with these low temperatures and have a cooling capacity up to $800 \mathrm{~W}$ per detector half. The cooling system, inside the LHCb acceptance, must be a low mass system. To avoid risk of leaks into the vacuum, no connectors can be used. We therefore use failsafe orbital welds and vacuum brazing connections.

\section{The VELO Thermal Control System (VTCS)}

The VELO Thermal Control System (VTCS) [3] meets all these constraints. The detector waste heat is transported through a cascade of three hydraulic systems. The detector is connected to the tertiary system, which is a mechanically pumped $\mathrm{CO}_{2}$ loop, cooled by a secondary freon chiller. The secondary chiller on its turn is cooled by the primary system based on chilled water. Figure 2 shows a block scheme of this cascade. 


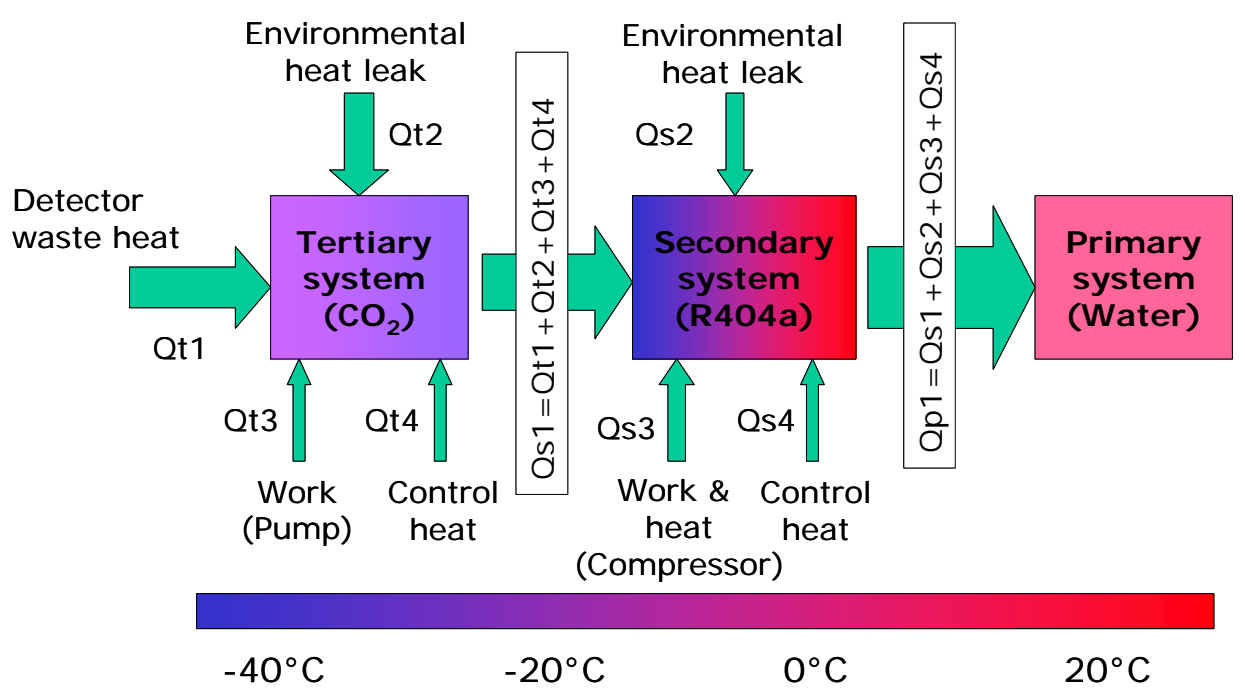

Fia 2: Block scheme and heat balance representation of the VTCS

\subsection{The tertiary $\mathrm{CO}_{2}$ system.}

The tertiary system uses a 2-Phase Accumulator Controlled Loop (2PACL) [4] method with $\mathrm{CO}_{2}$ as working fluid. A schematic overview and the thermodynamic principle can be found in Figure 3 and Figure 4 respectively. The numbers in parentheses in the following refer to the nodes in these figures.

The VTCS systems' pressure is regulated by the accumulator, a vessel always containing a saturated mixture of vapour and liquid $\mathrm{CO}_{2}$. The accumulator is parallel connected between the condenser and the pump at node 1 in Figure 3. The low hydraulic resistance between the evaporator outlet and the accumulator (node 10 to 1) assures that the pressure in the evaporator is close to the pressure in the accumulator. When in saturation this pressure therefore directly determines the temperature of the evaporator. In this way it is possible to control the evaporator temperature over a long distance without complex local actuators, making the distant evaporator completely passive. This approach greatly reduces the risk of failures in a hostile radiation area which is difficult to access.

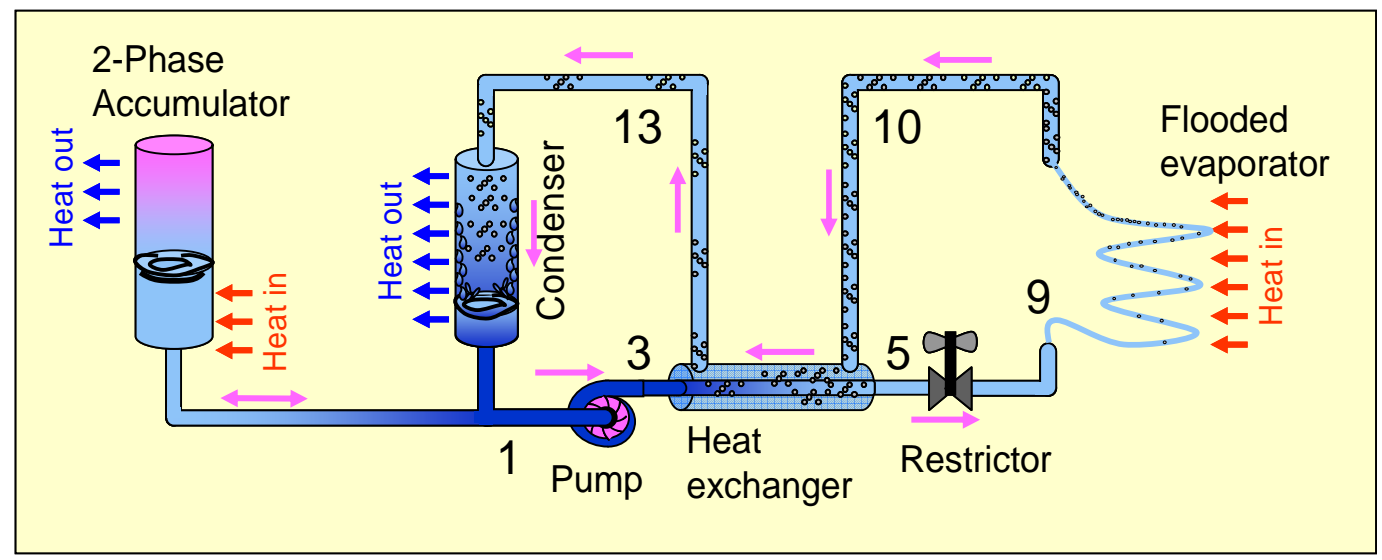

Fig. 3: Schematic overview of the $\mathrm{CO}_{2}$ system. 


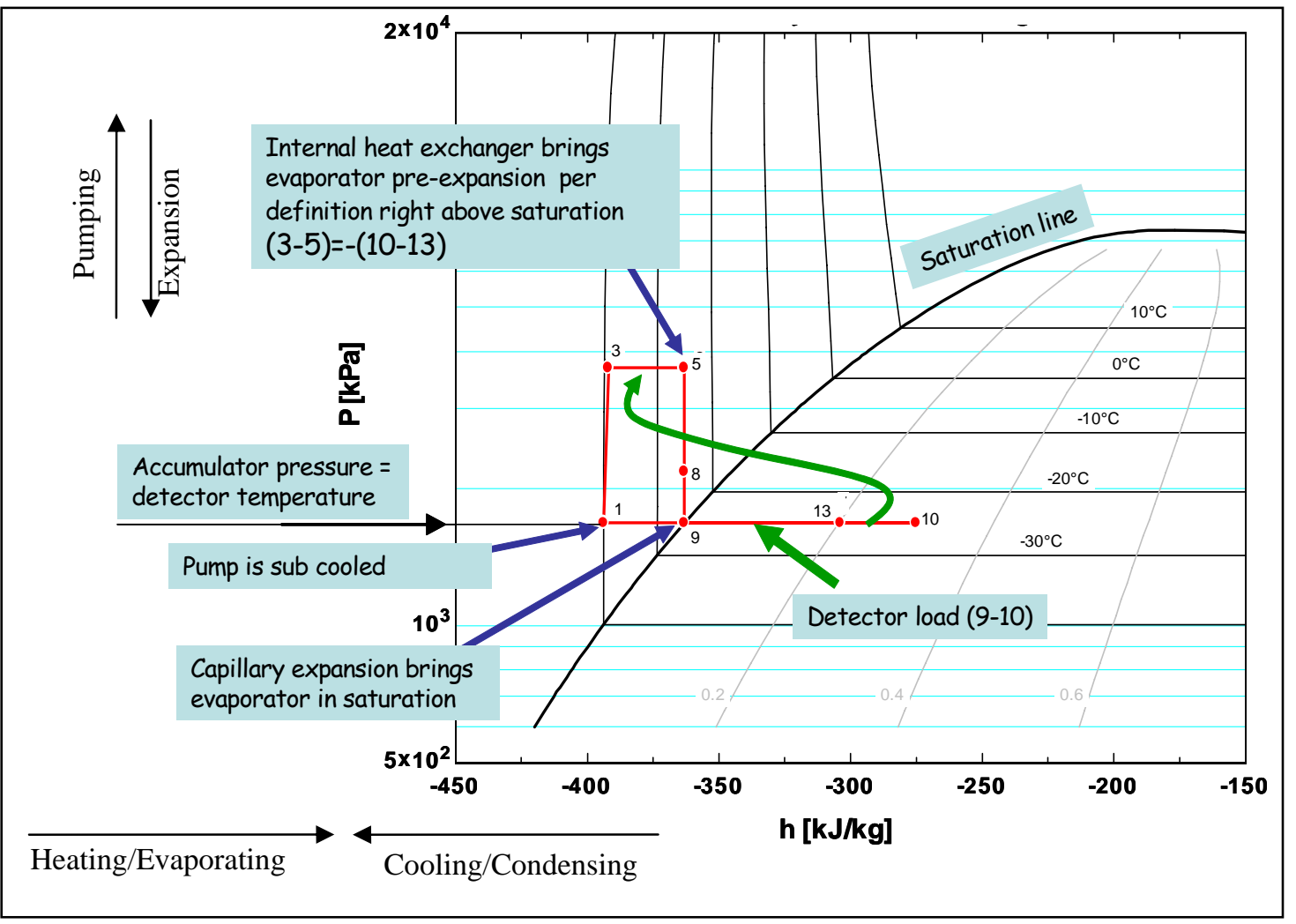

Fig 4: VTCS operation in the Pressure-Enthalpy diagram for $\mathrm{CO}_{2}$

In the VTCS-2PACL liquid carbon dioxide is mechanically pumped (node 1 to 3 ) to the evaporator by a membrane pump. The liquid goes to the detector through a $50 \mathrm{~m}$ long internal heat exchanger (node 3-5) where the liquid is heated up through the contact with the $\mathrm{CO}_{2}$ returning from the detector (node 10 to13). The liquid is then adiabatically expanded over restrictions (node 5 to 9). The expansion brings the $\mathrm{CO}_{2}$ from a sub-cooled liquid state ${ }^{1}$ at node 5 into saturation at node 9 before it enters the evaporator in the detector. The detector waste heat is removed by the latent heat of evaporation of the saturated $\mathrm{CO}_{2}$ (node 9-10). The liquid-vapour mixture having a typical vapour quality of 0.15 to 0.3 is returned through the return line of the heat exchanger (node 10-13). In this heat exchanger the mixture is already partly condensed by exchanging heat with the liquid of the inlet line. The mixture is then further condensed and sub-cooled by the freon chiller in the $\mathrm{CO}_{2}$ condenser (node 13 to 1 ). The sub-cooled liquid is fed back to the pump and the cycle is started again.

A useful feature of the 2PACL is the ability of turning the two-phase loop into a singlephase loop. Increasing the accumulator's pressure high enough will suppress any formation of vapour in the loop. This feature is a useful at start-up (see Section 2.3.1).

\footnotetext{
${ }^{1}$ Sub-cooled liquid is defined as a liquid which has a temperature a few degrees below its boiling point.
} 


\section{$2.2 \quad$ The VTCS design}

The cooling plant, containing the active and control components, is situated about $50 \mathrm{~m}$ from the passive $\mathrm{CO}_{2}$ evaporator. The evaporator and the cooling plant are linked through the transfer line. This gives the advantage that the control part is always accessible as it is situated behind the shielding wall and well protected from the hostile radiation environment. The $50 \mathrm{~m}$ transfer line is the internal heat exchanger between the liquid feed and the vapour return line. The liquid feed line lies inside the vapour return line. The cooling plant consists of the freon chiller filled with the refrigerant R507a and the active parts of the $\mathrm{CO}_{2}$ system.

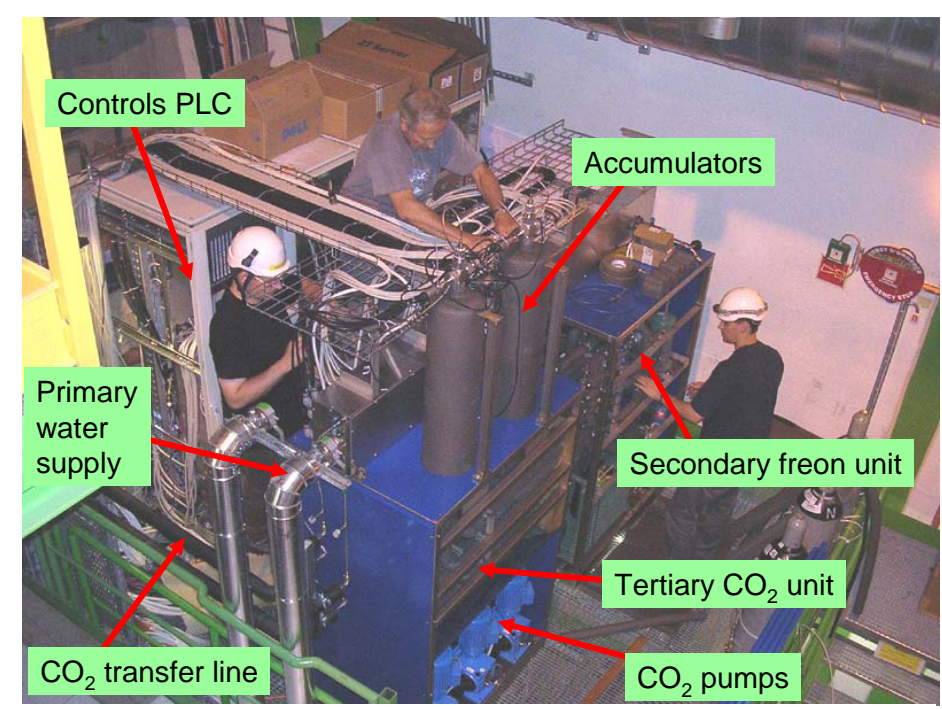

Fig.5: The Freon chiller rack, accumulators and the $\mathrm{CO}_{2}$ rack.

The chiller unit is a commercially engineered item, while the $\mathrm{CO}_{2}$ system is designed and built at Nikhef, Amsterdam. The $\mathrm{CO}_{2}$ unit contains the membrane pumps, the accumulators, all necessary valves and the plate condensers. The latter are commercially available brazed plate heat exchangers reinforced with steel end plates to withstand a maximum system pressure of 130 bar. A photograph of the cooling plant is shown in

Figure 5. It was installed in the LHCb experimental site during summer 2007 and entered a commissioning phase in the months after.

Redundancy is built in to assure the permanent cooling capacity. With three pumps the $\mathrm{CO}_{2}$ can always be pumped through the circuit. The freon chiller contains a water (main) and air (backup) cooled condenser. Each detector half is cooled by its own $\mathrm{CO}_{2}$ loop and is filled with about $11.5 \mathrm{~kg}$ of $\mathrm{CO}_{2}$. The entire system is controlled by a Siemens 400 series PLC.

\subsubsection{The accumulator}

The accumulator is a vessel always containing a saturated mixture of vapour and liquid $\mathrm{CO}_{2}$. With the accumulator the system pressure and hence saturation temperature can be increased or decreased. The accumulator contains a thermo siphon heater of $1 \mathrm{~kW}$ which allows for pressure increase by evaporation of the liquid in the mixture. Conversely, a cooling spiral fed by the freon chiller permits the mixture to condense and hence a pressure decrease. The cooling capacity is as well in the order of $1 \mathrm{~kW}$. The maximum operating pressure of the accumulator is 130 bar while it has volume of 14.2 liter. This vessel falls under the European directive for high pressure vessels and was thus CE certified. 


\subsubsection{The evaporator}

The passive evaporator is situated inside the vacuum of the detector. The liquid flow is distributed through restrictions over parallel cooling connections for each detector half. The connections are obtained by vacuum brazing and orbital welding. A photograph and
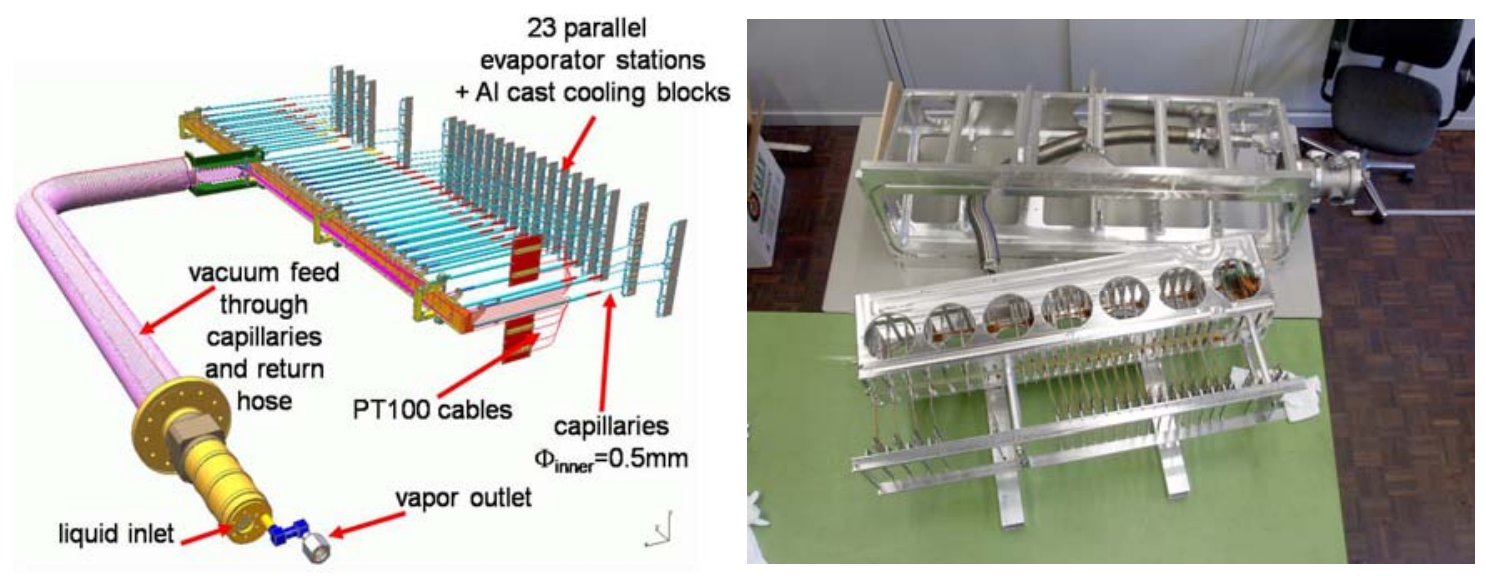

Fig. 6: The evaporator (left side) being installed in the VELO detector half (right side)

a design impression of the evaporator body are shown in Figure 6. The distribution capillaries have an inner diameter of $0.5 \mathrm{~mm}$ and bring the $\mathrm{CO}_{2}$ to each of the module stations. All the stations are equipped with aluminium cast cooling blocks, also called cooling cookies. In this process the stainless steel evaporator capillaries are imbedded in the aluminium, by melting the aluminium around the capillaries. The flat side of the cooling cookies are attached to the VELO modules by Thermflow T710 [5] for a good thermal connection. The return capillaries are connected through a manifold to the return line. The temperature of the cooling cookies and further system temperatures are monitored by 4 wire PT100 sensors.

\subsection{The VTCS performance}

\subsubsection{Operating the VTCS}

A simulation model was made to study the thermal equilibria of the different operational states. Figure 7 shows the simulation results of the various situations from warm startup to cold operation. During commissioning the start-up phase was verified. This corresponding data are shown in Figure 8. The different operational states are indicated in these figures by letters A to E. Prior to start-up the temperature in the loop is equal to the environmental temperature. This is the situation in the pressure-

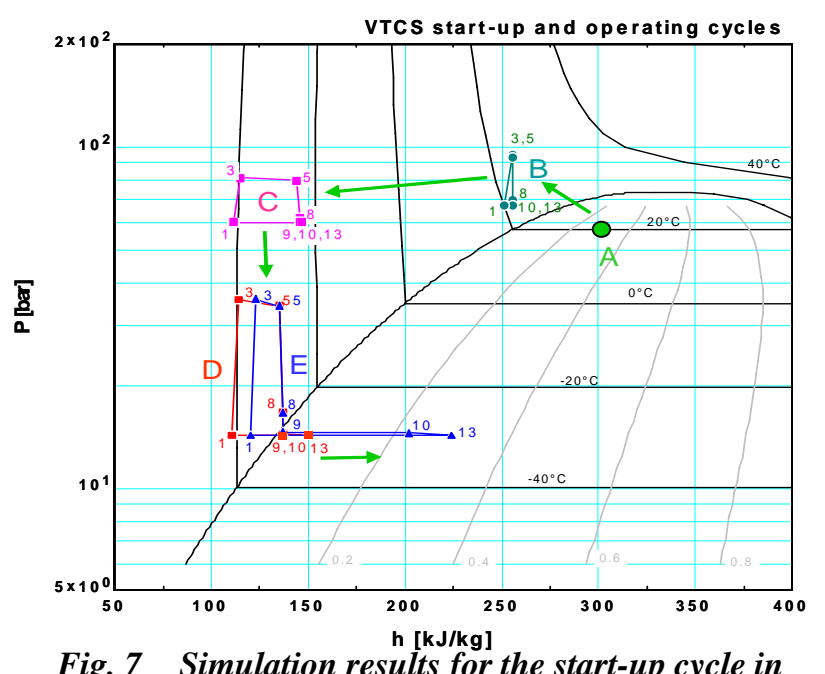

Fig. 7 Simulation results for the start-up cycle in the pressure-enthalpy diagram 
enthalpy diagram along the $20^{\circ} \mathrm{C}$ isotherm in the two-phase region (A). It is unknown where the liquid or vapour is situated. To prime the system the accumulator is heated. The increasing pressure will cause the loop to be filled with liquid. The vapour in the

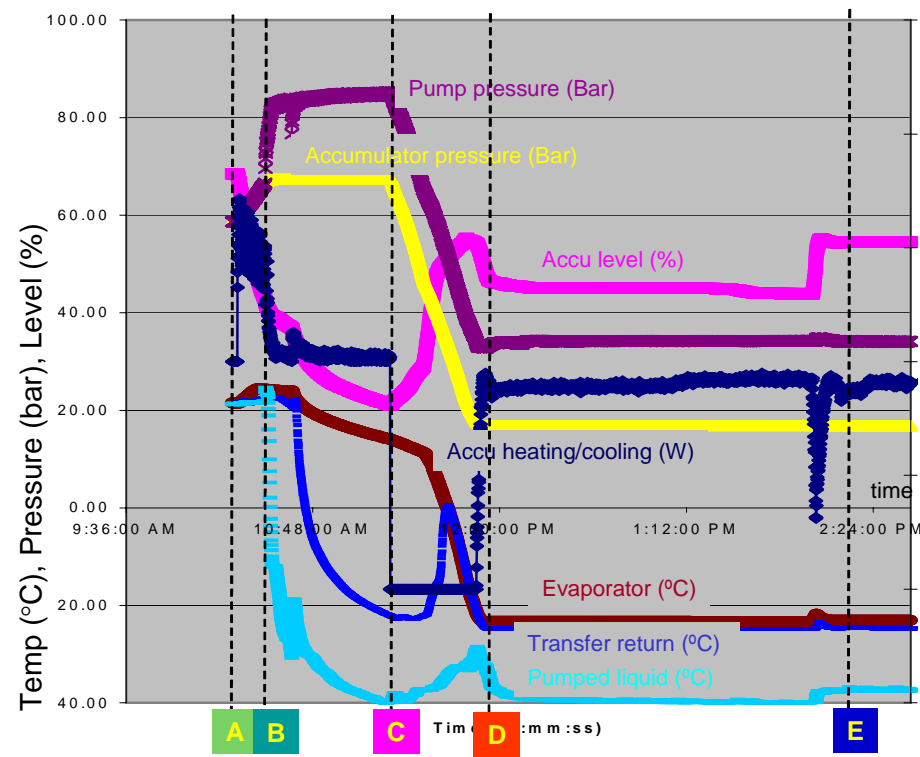

Fig.8 Data taken to illustrate the VTCS start-up loop condenses; the liquid is supplied by the accumulator. Once the loop is flooded the pump can be switched on and liquid $\mathrm{CO}_{2}$ of room temperature is now pumped around in the loop (B). Next the cooling by the freon chiller is switched on and the $\mathrm{CO}_{2}$ liquid is cooled down (C). The pumped liquid will be at about $-40^{\circ} \mathrm{C}$, while the evaporator stays relatively warm when in liquid mode. The thermal difference between pumped liquid (node 3 in Figure 3) and the returned liquid (node 13) is mainly due to the heat leak of the transfer line. Once cold liquid is circulating through the evaporator, the accumulator pressure and hence temperature can be lowered to the required set-point; this is a saturation temperature of $-30^{\circ} \mathrm{C}$ in Figure 7 and $-25^{\circ} \mathrm{C}$ in Figure 8. During accumulator cool-down the evaporator is cooled simultaneously. In Figure 8 the accumulator cooling is expressed as a negative heating power. During this cool down, the accumulator liquid level is increasing due to the increased vapour volume in the system.

In situation (D) the accumulator has reached its desired set-point temperature. The evaporator is saturated and its temperature is dictated by the accumulator, even though there is no heat to absorb. The VTCS is now ready for a detector power-on. The complete start-up procedure is implemented in the PLC and just requires a one push-button action. It takes about 2 hours to go from room temperature to a set-point temperature of $-25^{\circ} \mathrm{C}$.

\subsubsection{VTCS evaporator performance}

Once situation $\mathrm{D}$ is achieved the detector can be switched on. The powered-on situation is indicated in E of figures 7 and 8. Figure 9 zooms in on situation $\mathrm{E}$ of Figure 8. The shown test results were not obtained by the detector evaporator but by a parallel mounted evaporator containing an electrical heater. Situation $\mathrm{E}$ in figures 8 and 9 shows the behaviour when a load of $600 \mathrm{~W}$ is applied to one half. This represents 1.5 times the expected detector load per half. 
Before the load is applied, we observe that the temperatures at the evaporator and at the accumulator are stable at $-23.4^{\circ} \mathrm{C}$ and $-24.8^{\circ} \mathrm{C}$ respectively. The difference of $1.4^{\circ} \mathrm{C}$ between the evaporator and the accumulator is the evaporator offset which is caused by the flow resistance in the return line. After the load is applied, we observe a temporary increase in evaporator and accumulator pressure and hence a temperature increase of about $1.5^{\circ} \mathrm{C}$. This increase is caused by

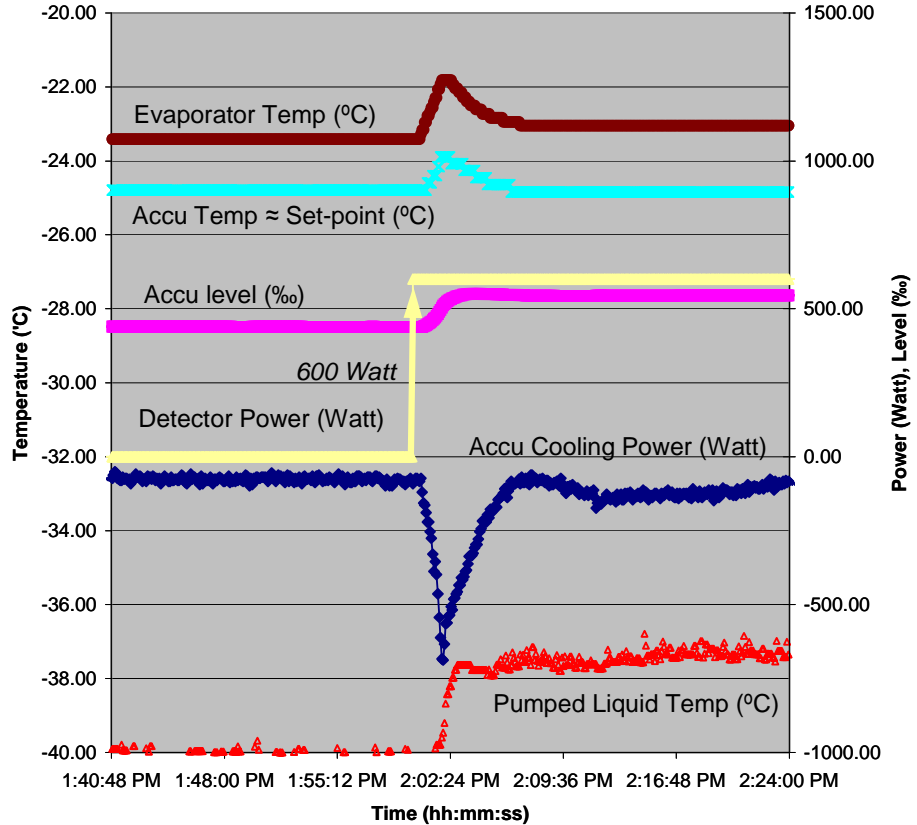

Fig.9 Data taken to illustrate the evaporator performance the increasing amount of vapour in the return line. Cooling down the accumulator will cause the pressure to go down with an increased accumulator level as result. Within 7 minutes the system is stabilised again and the evaporator temperature has increased from $-23.4^{\circ} \mathrm{C}$ to $-23.0^{\circ} \mathrm{C}$. The increased evaporator offset of $0.4^{\circ} \mathrm{C}$ is due to the increase of vapour in the return line, because vapour has more flow resistance compared to liquid. All these results are within specifications of the system.

\subsubsection{Evaporator performance in the lab}

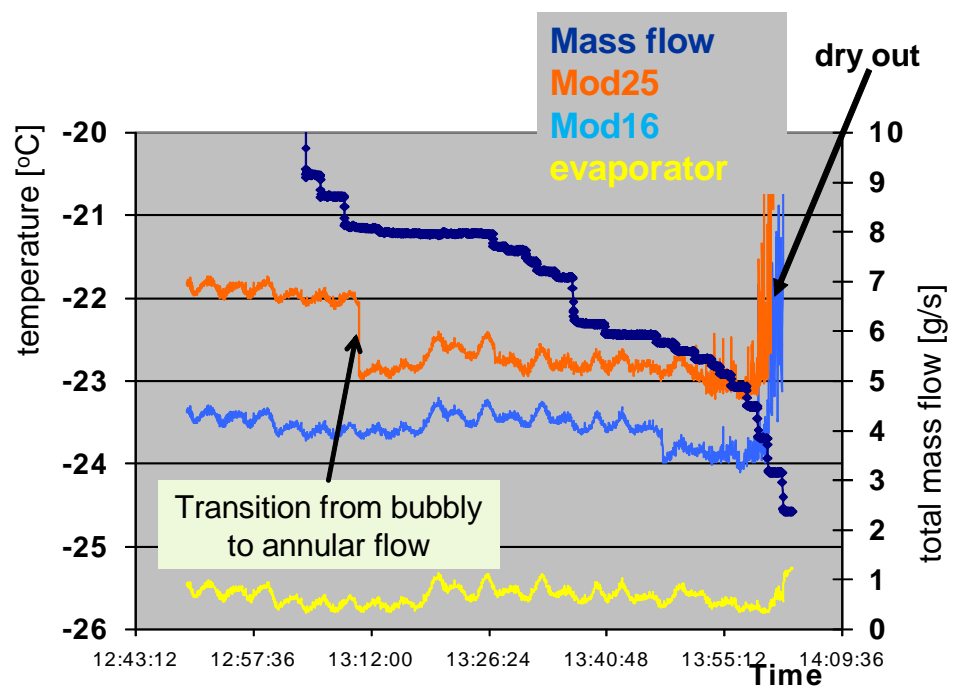

Fig.10: Performance of the evaporator at reducing flow, heat load on the module was $30 \mathrm{~W}$.
Both evaporators were fully characterised in the lab after being assembled. One of the phenomena that have been investigated is dryout. This happens when the mass flow is so low (and hence the vapour quality so high) that no liquid film on the capillary walls is formed any longer. The heat transfer is strongly reduced resulting in strongly increasing cooling block temperatures.

The characterisation tests of the evaporators demonstrated that the 
typical mass flow at which this phenomenon occurs is 5-6 g/s, as can be seen in Figure 10. The nominal flow for our evaporator is $12 \mathrm{~g} / \mathrm{s}$. Hence we are well above this limit.

\section{Module cooling performance}

Once the modules were installed on the detector bases their electrical [6] and thermal performance [7] was checked in the lab. A small scale cooling system blowing liquid $\mathrm{CO}_{2}$ through the evaporator tubes at $-25^{\circ} \mathrm{C}$ was used during these tests. All modules are equipped with NTCs to monitor the thermal performance. Per module side 2 NTCs are installed, NTC0 close to the cooling cookie region and NTC1 close to the silicon.
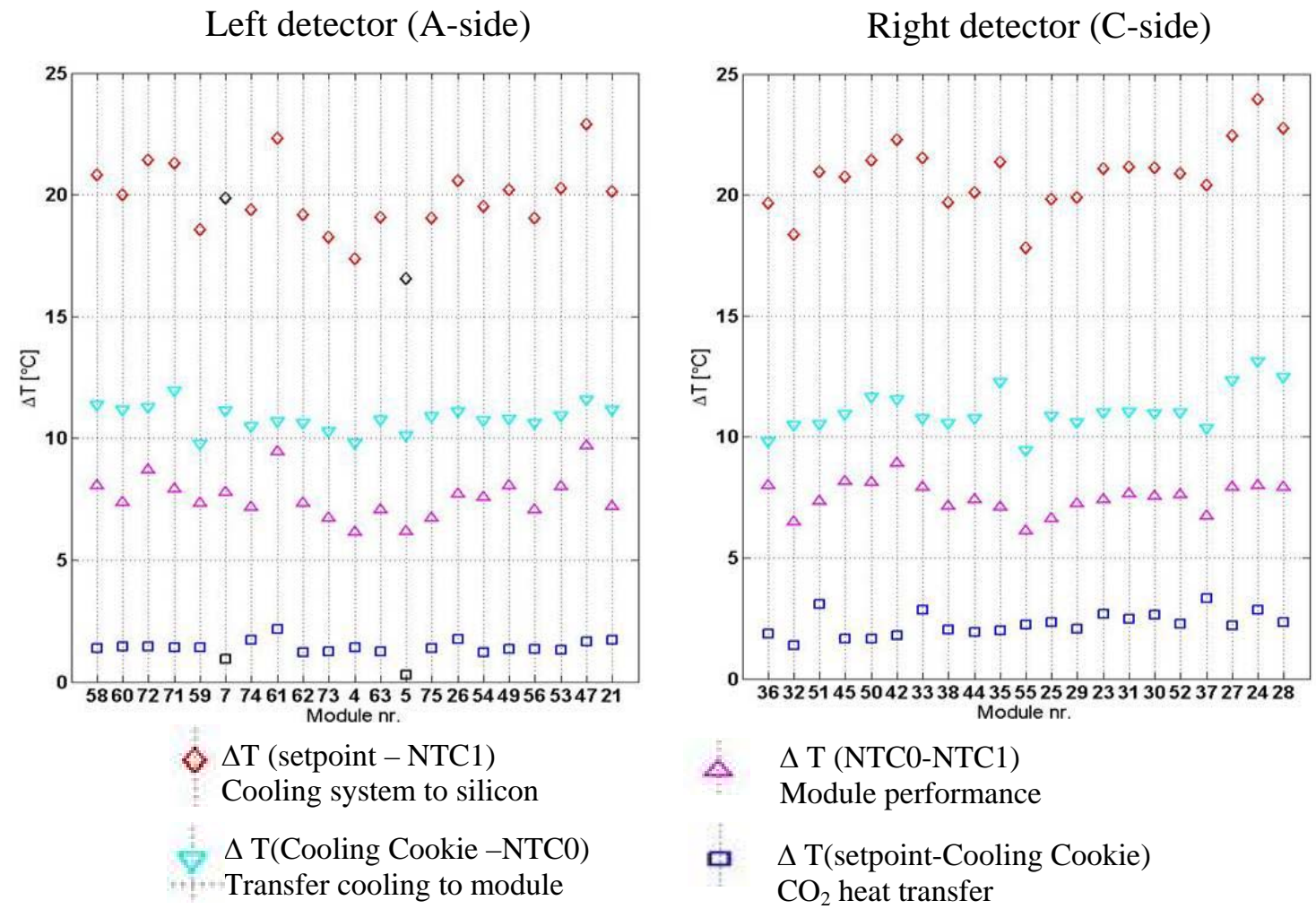

Fig. 11: Thermal performance of the VELO modules for both halves.

The results of the measurements for 42 modules are shown in Figure 11. Several differences in temperature are plotted against the module number. The difference in temperature between the silicon and the set-point temperature is about $20^{\circ} \mathrm{C}$ (diamonds in Figure 11). This total difference is the result of several contributions, temperature difference (i) from the $\mathrm{CO} 2$ system to the cooling cookie (blue squares), (ii) due to the coupling of the cooling interface to the TPG ${ }^{2}$ (downside triangles), (iii) along the TPG of the module (upside triangles) up to the silicon. The largest contribution is the

\footnotetext{
${ }^{2}$ TPG stands for Thermo Pyrolythic Graphite and is used as a thermal highway to improve the module heat conductivity.
} 
coupling of the cooling cookie interface to the TPG. An overview of the temperatures reached on the silicon with a set-point temperature of $-25^{\circ} \mathrm{C}$ is given in Table 1 .

\begin{tabular}{|l|c|c|}
\hline T on silicon $\left({ }^{\circ} \mathrm{C}\right)$ & Left/A Half & Right/C half \\
\hline Mean & $-4.2 \pm 1.4$ & $-5.2 \pm 1.5$ \\
\hline Min. & -7.2 & -8.4 \\
\hline Max. & -1.0 & -2.1 \\
\hline
\end{tabular}

Table 1: Summary of the thermal performance of the VELO modules.

The measurement conditions in the lab are not exactly the same as in the final system. The vacuum quality is a bit lower $\left(10^{-3}\right.$ mbar instead of the expected $\left.10^{-4} \mathrm{mbar}\right)$ and all the modules are not cooled and operated (heat load put on the modules) simultaneously.

\section{Conclusions}

Mid July 2007 the VELO Thermal Control System was installed in the experimental site. During subsequent commissioning the performance of the system was verified. The results found are matching the design parameters of the system. Set-point temperatures were tested down to $-25^{\circ} \mathrm{C}$ until now and the system proved stable operation. Detector loads up to $800 \mathrm{~W} /$ half were tested. The redundancy to keep the silicon at all times below $0^{\circ} \mathrm{C}$ is built in and testing has started. The thermal performance of the modules was verified and at a cooling temperature of $-25^{\circ} \mathrm{C}$ all modules are below the required $0^{\circ} \mathrm{C}$. The implementation of the PLC control proved successful and main operation of the system just requires a one push-button action. We are looking forward to enter the final commissioning phase with the VELO detector installed.

\section{References}

[1] LHCb Coll., LHCb Reoptimized Detector design and performance, CERN/LHCC 2003-30

[2] LHCb Coll., The LHCb VELO technical design report, CERN/LHCC 2001-0011

[3] B. Verlaat, Thermal performance Testing of the VTCS evaporator and VELO module, NIKHEF EN05-01, 2005.

[4] B. Verlaat, Controlling a 2-phase $\mathrm{CO}_{2}$ loop using a 2-phase accumulator, International Conference of Refrigeration 2007, Beijing, ICR07-B2-1565

[5] http://www.chomerics.com/products/documents/TB77page.pdf

[6] F. Da Cunha Marinho, Quality assurance tests of the LHCb VELO modules, These proceedings.

[7] T. Theunissen and A. Van Lysebetten, Thermal performance of the VELO modules, LHCb note, to be published 